\title{
Colon Cancer with Ovarian Metastasis during Pregnancy: A Case Report
}

Min Yin ${ }^{1 *}$ and Aiping Chen ${ }^{2}$

${ }^{1}$ Qingdao University, Qingdao, Shandong Province, China

${ }^{2}$ The Affiliated Hospital of Qingdao University Qingdao, Shandong Province, China

*Corresponding author: Min Yin, Qingdao University, Qingdao, Shandong Province, China, Tel: +8615621456898; E-mail: 1239096840@qq.com

Received Date: Oct 01, 2018; Accepted Date: Oct 23, 2018; Published Date: Oct 31, 2018

Copyright: @ 2018 Yin M, et al. This is an open-access article distributed under the terms of the Creative Commons Attribution License, which permits unrestricted use, distribution, and reproduction in any medium, provided the original author and source are credited.

\begin{abstract}
Colorectal cancer with ovarian metastasis is a rare occurrence, especially during pregnancy. A 43-year-old pregnant Chinese woman at 36 weeks gestation presented with a big adnexal mass and constipation. Elective cesarean section was performed, and a normal infant was delivered. Intraoperative frozen section pathology revealed differentiated adenocarcinoma with necrosis in the right ovarian tumor. In the further exploration of abdominal cavity, sigmoid colon cancer was diagnosed. High clinical suspicion is needed to diagnose colorectal cancer during pregnancy, which can present with symptoms that are common during pregnancy such as constipation. This can make the diagnosis of colorectal cancer during pregnancy difficult. Once diagnosed, surgery can be considered if resectable, taking into account gestational age. Fetal safety is a major consideration during treatment.
\end{abstract}

Keywords: Colon cancer; Ovarian metastasis; Pregnancy; Constipation; Cancer in pregnancy

\section{Introduction}

The incidence of cancer during pregnancy ranges from $0.07 \%$ to $0.1 \%$. Colorectal cancer is one of the eight most frequent malignant neoplasms in pregnancy [1]. Ovary is a one of the common metastasis sites of malignant tumor. In normal population, the probability of metastasis of colon cancer to ovary is $3 \%$ to $8 \%$ [2]. Constipation is a common symptom experienced during pregnancy. Other common presenting symptoms of colorectal cancer include abdominal pain, vomiting, and anemia; all of which are commonly seen in pregnancy. This is a major reason why colorectal cancer may be diagnosed later in pregnancy. Here, we report a case of colorectal cancer with ovarian metastasis who was presented with a big adnexal mass and constipation. The purpose of this case report is to increase awareness about colorectal cancer in pregnant women given the similarity of symptoms between normal pregnancy and cancer.

\section{Case Report}

The patient was a 43-year-old woman (gravid 5, para 1) at 36 weeks gestation by last menstrual period who was referred with the complaints of big adnexal mass and constipation. She did her prenatal examination regularly in their local hospital. She presented abdominal distension especially the right lower abdomen, and constipation for over one month. She denied blood per rectum. Ultrasonography in our hospital revealed a $15.5 \times 10.3 \times 7.9 \mathrm{~cm}$ cystic-solid mass with mixed echo on right side of the gravid uterus with a normal fetus. The laboratory examination showed carbohydrate antigen $12567.45 \mathrm{U} / \mathrm{ml}$, carbohydrate antigen $19-9130.00 \mathrm{U} / \mathrm{ml}$ and carcinoembryonic antigen $6.86 \mathrm{ng} / \mathrm{ml}$. Physical examination revealed neither vaginal discharge nor decreased fetal movement. She has a history of hysteromyoma for two years without any treatment and the patient did not mention any positive family history of cancer when she was asked. According to the ultrasonography and tumor markers, the big adnexal mass was highly suspected ovarian originated. In view of the risk of tumor rupture during transvaginal delivery, a cesarean section and exploratory laparotomy were recommended. Elective cesarean section was done at 36 weeks of gestation, and a normal infant weighing $2940 \mathrm{~g}$ was delivered. Intraoperative frozen section pathology revealed differentiated adenocarcinoma with necrosis in the right ovarian tumor. , a tumor about $4 \mathrm{~cm}$ was observed in the middle segment of the sigmoid colon, penetrating the serosa, and scattered in the uterine and rectal pits. An extensive cytoreduction surgery was performed including hysterectomy, bilateral salpingo-oophorectomy, pelvic and abdominal aortic lymph node dissection, larger omentectomy and appendectomy. Sigmoid colon resection and colostomy was performed. The total operating time was 8 hours, and the blood loss was estimated at $1000 \mathrm{~mL}$, with 1.5 units of packed red blood cells administered during the operation (Figures 1-3).

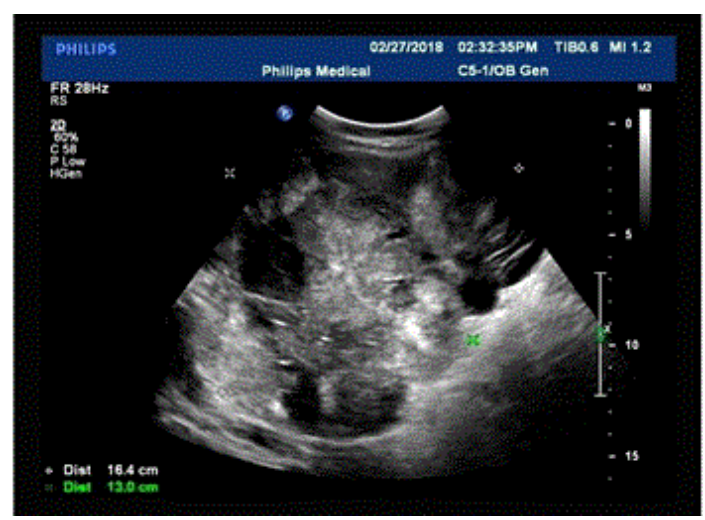

Figure 1: Ultrasonography revealed a $15.5 \times 10.3 \times 7.9 \mathrm{~cm}$ cysticsolid mass with mixed echo.

Postoperative pathology revealed differentiated adenocarcinoma with necrosis in the right ovarian tumor. The immunohistochemistry 
result showed CK7 (-), CK20 (+), CDX-2 (+), Villin (+), ER (-), PR (-), Pax-8 (-), p16 (+), p53 (-), WT-1 (-), Vimentin (-), 60\% Ki-67 (+), HNF1b (-). Combined with history and immunohistochemistry, it is consistent with metastasis originated from colon. Adenocarcinoma of the sigmoid colon is moderately differentiated, and metastasis is seen in the intestinal lymph nodes (8/16). Postoperative PET / CT was performed and revealed both lung and liver metastasis. Further treatment was carried out in the oncology department of our hospital. So far, five cycles of "XELOX" chemotherapy have been performed. The multiple nodules in both lungs and liver were slightly smaller than before.

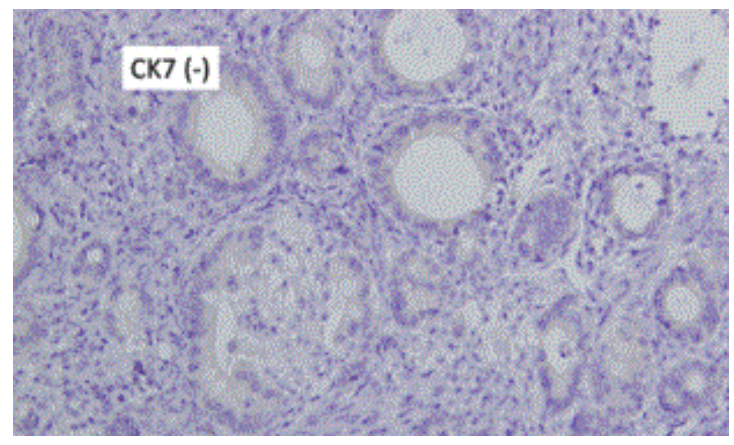

Figure 2: Immunohistochemical staining: negative for CK7.

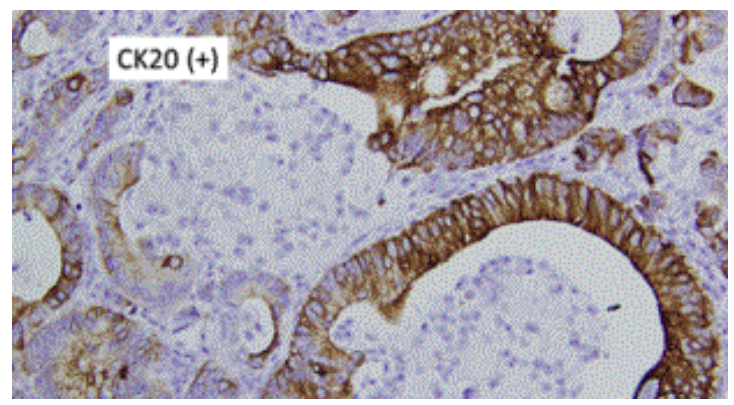

Figure 3: Immunohistochemical staining: positive for CK20.

\section{Discussion}

The incidence of cancer in pregnancy ranges from $0.07 \%$ to $0.1 \%$, with the most common types being cervical cancer, breast cancer, melanoma and leukemia. Colorectal cancer is one of the eight most frequent malignancies during pregnancy [1]. Ovarian metastasis was reported to be likely in colon cancer associated with pregnancy. In normal population, the probability of colon cancer metastasis to ovary is $3 \%-8 \%$ [2]. However, the risk of ovarian metastases in pregnancy with colon cancer is $25 \%$ [3], which are associated with poor prognosis.

The clinical manifestations of colorectal cancer in pregnancy are similar to those of non-pregnant women. And its common symptoms including constipation, abdominal pain, anemia and altered bowel movements. Constipation is a common symptom experienced during pregnancy; however, it is also one of the earliest symptoms of colorectal cancer. Because these symptoms are generally found in normal pregnancy, most of the cases can be easily missed and are diagnosed in advanced stages. In this case, the patient's tumor was located in the middle sigmoid colon, without rectal bleeding. Constipation occurred more than one month ago, mistaken for the normal symptom in late pregnancy due to fetal head compression, being one of the main reasons for this patient's delayed diagnosis until near term labor. This allows more spread of cancer and makes it difficult to treat.

Treatment of colorectal cancer in pregnancy is another challenging issue. There are several factors to consider when planning management: location of the tumor, gestational age, the stage of the tumor, complications of tumor or pregnancy, and the patient's decision. chemotherapy is mainly determined by the patients. The risk and benefits should be discussed with the patient [4]. The current recommendation is that patients who undergo surgery in early pregnancy can be treated with chemotherapy in the third trimester. If diagnosed in late pregnancy, chemotherapy can be used after delivery [5]. Generally, in the first 20 weeks of pregnancy, treatment delay can lead to disease progression and compromise the mother's life; therefore, pregnancy would be terminated and early cancer treatment should be started. In the second 20 weeks of pregnancy, surgery can be delayed for saving the fetus, until fetal lung maturation is established $[6,7]$. As for the mode of delivery, it is not affected by cancer, with the exception of a cesarean section owing to a distal tumour obstructing the birth canal or anterior rectal wall carcinoma [4]. If cesarean section is needed for obstetric reasons, tumors can be removed simultaneously. In this case, given the fact that there is possibility of mass rupture and intra-abdominal hemorrhage during vaginal delivery, cesarean section was selected and exploratory surgery was performed. The patient was diagnosed during cesarean section, and the tumor had spread to ovary, peritoneum, omentum, rectum and uterine pit.

In summary, colorectal cancer is a rare but devastating event during pregnancy. And it can be masked by the symptoms that can be attributed to normal pregnancy. Therefore, the clinical symptoms and signs that cannot be explained by pregnancy alone should be paid enough attention by clinicians, especially to the mass of progressive growth. Other examinations like endoscopy, tumor markers and magnetic resonance imaging should be performed as early as possible if necessary, in order to discover malignant tumors at early stage and avoid missing the best time of treatment.

\section{Acknowledgments}

The authors would like to acknowledge the gynaecology and obatetrics teams in The Affiliated Hospitalof Qingdao University, Qingdao, Shandong Province, China. We also thank Bai Yiqi for editorial assistance and emotional encouragements.

\section{Disclosure}

This study was not funded. The authors declare that they have no conflict of interest.

\section{References}

1. Doll DC, Ringenberg QS, Yarbro JW (1988) Management of cancer during pregnancy. Arch Intern Med 148: 2058-2064.

2. Jr KLF, Ray JE, Overby I (1973) Ovarian metastases from colorectal carcinoma. Dis Colon Rectum 16: 305-311.

3. Matsuyama T, Tsukamoto N, Matsukuma K, Kamura T, Kaku T, et al. (1989) Malignant ovarian tumors associated with pregnancy: report of six cases. Int J Gynaecol Obstet 28: 61-66. 
Citation: Yin M, Chen A (2018) Colon Cancer with Ovarian Metastasis during Pregnancy: A Case Report. Med Rep Case Stud 3: 168. doi: $10.4172 / 2572-5130.1000168$

Page 3 of 3

4. Yaghoobi M, Koren G, Nulman I (2009) Challenges to diagnosing colorectal cancer during pregnancy. Can Fam Physician 55: 881-885.

5. Cappell MS (2003) Colon cancer during pregnancy. Gastroenterol Clin North Am 32: 341-383.

6. Berveiller P, Carbonne B, Mir O (2014) Cancer and pregnancy: an overview for obstetricians and gynecologists. Am J Obstet Gynecol 211: 82.
7. Peccatori FA, Azim HA, Orecchia R, Hoekstra HJ, Pavlidis N, et al. (2013) Cancer, pregnancy and fertility: ESMO Clinical Practice Guidelines for diagnosis, treatment and follow-up. Ann Oncol 24: 160-170. 